\section{Commentary: Adjuvant chemotherapy after trimodal therapy for esophageal carcinoma: A bitter pill to swallow}

\author{
Valerie X. Du, BA, and Shawn S. Groth, MD, MS
}

Despite numerous contemporaneous advancements in oncology care, the 5-year survival rate for locally advanced esophageal cancer remains $30 \%$ to $50 \%$, even with aggressive multimodal therapy. In most centers in the United States, the current preferred treatment approach for locally advanced disease is concurrent neoadjuvant chemoradiation followed by esophagectomy. However, many patients have an incomplete primary tumor and/or nodal response from neoadjuvant therapy and are at risk for systemic and local failure despite an R0 esophagectomy. Although nodal response is an important prognostic factor ${ }^{1}$ and adjuvant chemotherapy may offer a survival advantage particularly for patients with residual nodal disease, ${ }^{2}$ there is a lack of direction from cancer treatment guidelines with regard to which patients are most likely to benefit from adjuvant systemic therapy. Indeed, for postesophagectomy nodepositive esophageal adenocarcinoma and squamous cell carcinoma patients who received neoadjuvant chemoradiation therapy, the National Comprehensive Cancer Network recommends surveillance until disease progression and offers no explicit recommendations for the role of adjuvant treatment. $^{3}$

Kandilis and colleagues ${ }^{4}$ assessed the influence of persistently positive nodal disease after neoadjuvant chemoradiation on survival for 509 esophageal adenocarcinoma

From the Division of Thoracic Surgery, Michael E. DeBakey Department of Surgery, Baylor College of Medicine, Houston, Tex.

Disclosures: The authors reported no conflicts of interest.

The Journal policy requires editors and reviewers to disclose conflicts of interest and to decline handling or reviewing manuscripts for which they may have a conflict of interest. The editors and reviewers of this article have no conflicts of interest.

Received for publication Dec 22, 2020; revisions received Dec 22, 2020; accepted for publication Dec 23, 2020; available ahead of print Jan 21, 2021.

Address for reprints: Shawn S. Groth, MD, MS, Division of Thoracic Surgery, Michael E. DeBakey Department of Surgery, One Baylor Plaza, BCM 390, Houston, TX 77030 (E-mail: Shawn.Groth@bcm.edu).

JTCVS Open 2021;5:150-1

2666-2736

Copyright $\odot 2020$ The Authors. Published by Elsevier Inc. on behalf of The American Association for Thoracic Surgery. This is an open access article under the CC BY-NCND license (http://creativecommons.org/licenses/by-nc-nd/4.0/).

https://doi.org/10.1016/j.xjon.2020.12.013

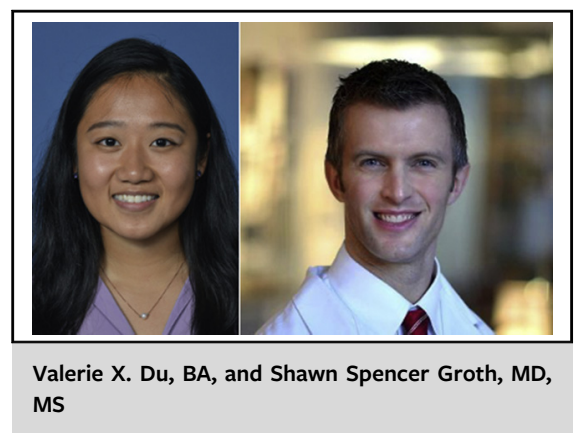

CENTRAL MESSAGE
In patients with esophageal can-
cer who have residual nodal dis-
ease in an esophagectomy
specimen following neoadjuvant
chemoradiation therapy, adju-
vant chemotherapy is linked to
improved survival.

patients who underwent esophagectomy at their institution over a 12 -year period. ${ }^{4}$ Similar to prior studies, they noted a $22 \%$ complete pathologic response rate for adenocarcinomas treated with neoadjuvant chemoradiation, highlighting the importance of at least careful consideration of esophagectomy independent of radiographic response to neoadjuvant therapy.

At a median follow-up of 60 months, patients who had persistently positive nodal disease had worse overall and disease-free survival and had higher rates of distant recurrence compared with patients who had a complete pathologic response or who had residual primary tumor but no nodal disease. Although the extent of lymphadenectomy did not influence survival, receipt of adjuvant therapy was associated with 13.6-month improvement in overall survival and a 10.8-month improvement in disease-free survival for those patients with residual nodal disease. The influence on disease-free survival was more profound for those with N2 or N3 compared with N1 disease. ${ }^{4}$

Although patients with persistently positive nodal disease may benefit from adjuvant therapy, most cannot tolerate it. Indeed, perioperative chemotherapy trials have taught us that only $40 \%$ to $50 \%$ of patients who had it as part of their initial treatment plan can tolerate a full course of adjuvant therapy. Those who are unable to complete a 
planned course of adjuvant systemic therapy are likely at an increased risk of systemic failure. Therefore, the goal of neoadjuvant therapy should be to optimize nodal response rates and convert node-positive disease to node-negative disease before proceeding with esophagectomy, yet doing so without subjecting patients to endless cycles of chemotherapy. One possible approach is to assess response midtreatment and alter the systemic therapy regimen if the clinical response is not favorable, a concept being studied in the Cancer and Leukemia Group B (Alliance) 80803 trial. ${ }^{5}$ Alternatively, patients who are nonresponders may benefit from biomarker-directed neoadjuvant targeted therapy or immunotherapy. Clinical trials to further evaluate these strategies are needed.

\section{References}

1. Groth SS, Burt BM, Farjah F, Smaglo BG, Sada YH, Sugarbaker DJ, et al. Prog nostic value of neoadjuvant treatment response in locally advanced esophageal adenocarcinoma. J Thorac Cardiovasc Surg. 2019;157:1682-93.e1.

2. Burt BM, Groth SS, Sada YH, Farjah F, Cornwell L, Sugarbaker DJ, et al. Utility of adjuvant chemotherapy after neoadjuvant chemoradiation and esophagectomy for esophageal cancer. Ann Surg. 2017;266:297-304.

3. The NCCN Clinical Practice Guidelines in Oncology Esophageal and Esophagogastric Junction Cancers (version 4.2020). Available at: http://www.NCCN.org Accessed December 15, 2020.

4. Kandilis A, Bravo Iniguez C, Khalil H, Mazzola E, Jaklitsch MT, Swanson SJ, et al. Residual lymph node disease and mortality following neoadjuvant chemoradiation and curative esophagectomy for distal esophageal adenocarcinoma. J Thorac Cardiovasc Surg Open. 2021;5:135-47.

5. Goodman KA, Niedzwiecki D, Hall N, Bekaii-Saab TS, Ye X, Meyers MO. Initial results of CALGB 80803 (Alliance): a randomized phase II trial of PET scandirected combined modality therapy for esophageal cancer. J Clin Oncol. 2017; 35(4 Suppl 1):1. 